This is an Accepted Manuscript of an article published by Taylor \& Francis in International Journal of Injury Control and Safety Promotion on 04 Jul 2017, available online: https://www.tandfonline.com/doi/full/10.1080/17457300.2017.134 $\underline{1931}$

Without prejudice to other rights expressly allowed by the copyright holders, this publication can be read, saved and printed for research, teaching and private study. Any other noncommercial and commercial uses are forbidden without the written permission of the copyright holders. 


\title{
Unsafe Cycling Behaviors and Near Crashes among Italian Cyclists
}

Víctor Marín Puchades, Luca Pietrantoni, Federico Fraboni, Marco De Angelis, and Gabriele Prati

\author{
Author Note
}

Department of Psychology, University of Bologna, Bologna, Italy.

Correspondence should be addressed to:

Professor Luca Pietrantoni, luca.pietrantoni@unibo.it; +39 0512091389

Other authors' details:

Dr Víctor Marín Puchades, victor.marinpuchade2@unibo.it

Dr Federico Fraboni, federico.fraboni3@unibo.it

Dr Marco De Angelis, marco.deangelis6@unibo.it

Professor Gabriele Prati, gabriele.prati@unibo.it

Authors' addresses:

Dipartimento di Psicologia, Via Berti Pichat 5, 40126, Bologna, Italy.

This work was supported by the European Commission under the Horizon 2020 Framework Programme of the European Union (2014-2020). Project XCYCLE contract number: 635975.

Word count: 4242 


\begin{abstract}
This study investigates the direct and indirect effect of three types of unsafe behaviors (i.e., errors, generic violations, and smartphone-specific violations) on the likelihood of near crashes and actual crashes among Italian cyclists. Basing on the Generic Error Modelling System (GEMS), we explored the impact of errors, generic violations, and smartphone-specific violations in predicting near crashes. We considered smartphone-specific violations as a different unsafe behaviors subtype that enhance the probability of committing errors, thus increasing the likelihood of being involved in near crashes. Furthermore, we hypothesized that near crashes will predict actual crashes. Results revealed that errors predicted near crashes, whereas generic and smartphone-specific violations did not. Path analysis showed that near crashes mediated the effect of errors on crashes. Moreover, smartphone-specific violations predicted crashes throughout its consecutive effects on errors and near crashes. Finally, we found gender differences in near crashes and crashes, but not in unsafe cycling behaviours, while age was positively correlated with crashes and negatively correlated with unsafe cycling behaviours. These findings contribute to deepen our understanding of the relationship between cyclists' unsafe behaviors, near crashes, and actual crashes. To our knowledge, the present study is the first that links errors to near crashes among cyclists.
\end{abstract}

Key words: Cycling behaviors, Smartphone-specific violations, Errors, Bicycle accidents, Road crashes

\title{
Introduction
}


In the last decades, the amount of research investigating cycling safety has dramatically increased. Such interest might be due to several reasons. To begin with, infrastructures are usually not designed to provide cyclists with safety conditions comparable to other road users (e.g., car drivers), therefore, their level of protection is considerably lower (Wegman, Zhang, \& Dijkstra, 2012). Furthermore, even though cyclists represent a small minority in comparison with motorized vehicles, they account for a relatively large proportion of fatalities (ERSO, 2015a). As a matter of fact, in 2013 there were 2017 cyclists' fatalities in the EU countries, which correspond to the $7.8 \%$ of all the road deaths, which emphasizes their vulnerability (ERSO, 2015b). All the above constitute key reasons or arguments for the need of attention and emphasis on the study of cycling safety.

Fatality trends and other safety outcomes (e.g., number of accidents) vary along different EU countries. In Italy, according to the Italian National Institute of Statistics annual report (ISTAT, 2015), 18055 cyclists were injured in traffic accidents in 2014. Cyclists' deaths accounted for 273 people that died within 30 days following the accident. The mortality index (deaths every 100 accidents) for cyclists is 1.42 , which is more than double compared to car users (0.67). In 2014 the number of cyclists' fatalities in Italy increased by $8.8 \%$ compared to the previous year. Older cyclists represent the category at higher risk, accounting for $42 \%$ of the total death. Amongst the injured, people aged from 34 to 54 years old are overrepresented (33\% of the total). To stress the importance of the present study, from 2001 to 2014 the Italian context has seen a reduction of cyclists' fatalities of the $25 \%$, compared with a decrease in car driver fatalities of the $61.2 \%$. Consistently, from 2004 to 2013 the reduction in cyclists' fatalities in EU reached the $32 \%$ versus a decrease of 22\% in Italy (ERSO, 2015b).

According to the Italian Country Overview report of 2015 (ERSO, 2015a), roads are particularly dangerous for cyclists because Italian car drivers generally display less road safety culture. Particularly, in relation to self-reported driving behavior, Italian car drivers admit to 
engage in dangerous behaviors such as too close following, speeding up to $20 \mathrm{~km} / \mathrm{h}$ over the limit in residential areas, and make/answer calls with handheld phone more frequently than the European average. Furthermore, concerning road safety attitudes, the probability of being checked for speeding and alcohol use is perceived as lower in Italy than in other European countries, and traffic rule enforcement is assessed as less effective than the EU average. Thus, based on the unique characteristics of the Italian context we decided to conduct the present study in Italy.

Many aspects hold importance when accounting for accident causation and injury severity. The Haddon matrix considers factors related to the environment (e.g., weather, road conditions), the vehicle and equipment, and the human (Runyan, 1998). The present paper focuses on human behavior, which has been identified as a key factor when exploring road crashes (Rowe, Roman, McKenna, Barker, \& Poulter, 2015). With reference to the study of road behavior, the Generic Error Modeling System (GEMS; Reason, 1990) has been widely applied across types of road users, either car drives (e.g., De Winter \& Dodou, 2010), motorcyclists (e.g., Sakashita, Senserrick, Lo, Boufous, Rome, \& Iver, 2014) and cyclists (e.g., Twisk, Commandeur, Vlakveld, Shope, \& Kok, 2015). GEMS allows for distinction of several types of behaviors and prediction of cycling safety outcomes (e.g., Twisk et al., 2015). The model identifies two categories of risk behaviors, namely 'errors' and 'violations', each governed by different psychological mechanisms. Errors have been defined as 'the failure of planned actions to achieve their intended consequences' (Reason, Manstead, Stradling, Baxter, \& Campbell, 1990, p. 1315). Thus, they involve unintentional deviations from safe practices and reflect inadequate skills (e.g., because of inexperience), or temporarily adverse states (e.g., because of fatigue) involving information processing. Violations, on the contrary, are 'deliberate deviations from those practices believed necessary to maintain the safe operation of a potentially hazardous system' (Reason et al., 1990, p. 1316), for instance, deliberately 
violating a red light. Therefore, they reflect a person's safety motivation, such as, a trade-off between risk and time lost. In addition, violations do not necessarily imply an infringement of some written rule, but they can also entail breaking an unofficial safety norm (Reason et al., 1990; Twisk et al., 2015).

Research studying unsafe cycling behavior from the GEMS perspective has focused on adolescent cycling behavior or particular types of violations. Feenstra, Ruiter, Schepers, Peters, and Kok (2011) found that, among adolescents in The Netherlands, boys were more prone to committing errors and violations, and so were older participants for exceptional violations. Moreover, they also reported that errors and violations, classified into common and exceptional, were related to near crashes. Errors were also associated with accident frequency and exceptional violations with accident severity, and no gender differences were found with regard to self-reported crashes frequency. Hollingworth, Harper, and Hamer (2015) found that always stopping at a red light (i.e., not committing a specific type of violation), was associated with lower risk of accident-related injury. Moreover, Twisk et al. (2015) proposed a mediation model in which risky behaviors were mediating the relationship between psychological determinants (e.g., opinions about alcohol, feeling responsible for one's actions) and safety outcomes (i.e., crashes and near crashes). The authors also found errors to be predicting crashes and near crashes in a subsample of 14 to 16 year-old students. Within this group, they reported several psychological determinants (i.e., opinions about alcohol, feeling of responsibility towards one's actions and competences in comparison to those of others) to be associated to risk behavior. In addition, they also found competences in comparison to others and feeling responsible for one's actions to have a direct effect on crashes and near crashes. Moreover, they also reported that in a group of 12 to 13 year-old students, hazard awareness, opinions about traffic rules, carelessness and feeling responsible for one's actions explained the $44 \%$ of the variance of risk behaviors. And these, comprising errors, dangerous play and lack of 
protective behavior, were found to be significantly associated with crashes along with age and bicycle exposure.

From the framework of the GEMS model, smartphone use whilst cycling should be considered a specific type of violation because, as stated above, it is a deliberate deviation from a safe practice. However, at the conceptual level there are reasons to consider it as a separate and unique type of violation. Terzano (2013) found differences in unsafe behaviors while performing secondary-tasks and cycling in comparison to those only riding a bicycle. In addition, several authors (DeWaard, Schepers, Ormel, \& Brookhuis, 2010; DeWaard, Edlinger, \& Brookhuis, 2011; DeWaard, Lewis-Ewans, Jelijs, Tucha, \& Brookhuis, 2014) have found that operating a smartphone led to reduced cyclist's visual detection and perception, posing a risk for cyclists. Therefore, such findings imply that this type of violations might have an effect on other sort of unsafe behaviors that rely on information processing, that is, they might be leading to increased error occurrence.

Several authors have pointed out the incidence of such behaviors. Goldenbeld, Houtenbos, Ehlers, and Waard (2012) reported that 70\% of their participants sometimes used a portable electronic device and one out of six of those that were under 35 used it during every trip. Moreover, DeWard, Westerhuis, and Lewis-Evans (2015), in an observational study, found a changing tendency from calling to operating phone screens while bicycling. They also reported that cyclists texting used to cycle further from curbs and used to gaze with less frequency at intersections when generally using a phone. In addition, Ichikawa and Nakahara (2008) found mobile phone usage to be associated with previous crashes and near crashes, thus being directly linked to critical safety outcomes. Moreover, Goldenbeld et al. (2012), using self-reported methods, found the use of portable electronic devices to be a risk factor for cyclists under 35 years old in The Hague (the Netherlands). Nevertheless, they also found different compensatory behaviors when using such devices, among which were paying better 
attention to traffic or wearing a helmet, thus, contradicting the findings by Terzano (2013) above mentioned.

All in all, even though the body of research of unsafe cycling behaviors is growing, there is still need for more research to further untangle how - and to what extent - errors and violations affect cycling safety outcomes, such as crashes and near crashes. Moreover, the main studies have focused on adolescent behavior (e.g., Feenstra, et al., 2011; Twisk, et al., 2015) or very specific violations (e.g., Hollingworth et al., 2015), and have investigated them in the context of The Netherlands (e.g., Feenstra, et al., 2011; Twisk, et al., 2015) and England (Hollingworth, et al., 2015). Therefore, there is need for further examination of such effects on adult population and in different countries, such as Italy which, to our knowledge, lacks scientific research on risky cycling behaviors. In addition, since previous research on other road users suggests that there might be age and gender differences in unsafe behaviors (e.g., Turner and McClure, 2003), we intend to explore such differences in our study. Furthermore, given the high frequency of smartphone use whilst cycling and the contradictory findings on whether it leads to more unsafe behaviors or to compensatory ones, there is need for clarification of such relationships. For this reason, we consider it is appropriate and consistent with the previously stated to deem smartphone-specific violations as qualitatively different from more generic violations, such as cycling against the traffic flow or drunk driving. Moreover, as previous research showed, this type of smartphone-specific violations might contribute to more errors, whereas this is not implied or required for more generic violations.

Based on the previously reported findings and the stated need for more research, we establish a hypothesized path model in which errors (hypothesis 1), generic violations (hypothesis 2) and smartphone-specific violations (hypothesis 3) will be positively associated with near crashes. Moreover, given the previously reported findings implying that performance 
of secondary-tasks while cycling leads to unsafe behaviors relying on information processing, we hypothesize that smartphone-specific violations will predict errors (hypothesis 4).

In the Safety Pyramid model, near misses comprise the lower part of the pyramid, while accidents are at the pinnacle (e.g., Phimister, Oktem, Kleindorfer, \& Kunreuther, 2003). Nearmisses has been used both to predict accidents and to limit accidents in a broad spectrum of industries, such as rail and air transport sector, medicine, and chemical process industry (e.g., Jeffs, Berta, Lingard, \& Baker, 2012; Jones, Kirchsteiger, \& Bjerke, 1999; Phimister et al., 2003; Saleh, Saltmarsh, Favarò, \& Brevault, 2013; Wright, \& Van der Schaaf, 2004). However, in the road safety literature, most studies conceptualized near misses as an outcome in combination with accidents. Thus, although there have been investigations that included nearmisses, these data were not used to predict the likelihood of crashes. Indeed, only limited evidence has been presented that investigates the association between near misses and actual driving accidents. One example is the study of Powell et al. (2007), in which it was found that near-miss sleepy driving accidents predict self-reported actual driving accidents. To address this research gap, we hypothesized that near crashes will predict actual crashes (hypothesis 5).

In a nutshell, we have hypothesized a model (see Figure 1) in which errors, generic violations, and smartphone-specific violations predict near crashes. In turn, near crashes were hypothesized to predict actual crashes. Together, the two types of relationship specify a model in which the three types of risky behaviors (i.e., errors, generic violations, and smartphonespecific violations) indirectly increase the likelihood of actual crashes by increasing the likelihood of occurrence of near crashes. Therefore, we hypothesized that near crashes will mediate the effect of the three types of unsafe behaviors on actual crashes (hypothesis 6). Moreover, we have also proposed that smartphone-specific violations will enhance the probability of committing errors, and this, at the same time will increase the likelihood of being involved in near crashes. In addition, since we have also posed that crashes will be predicted 
by near crashes, we hypothesize a serial mediation model in which errors will mediate the effect of smartphone-specific violations on near crashes, and these will act as a mediator between errors and the occurrence of crashes (hypothesis 7). The hypothetical model for this study is presented in Figure 1.

\section{[Insert Figure 1]}

\section{Method}

\section{Procedure}

Data were collected from December 10, 2015 to February 29, 2016 through a selfreported online questionnaire. In order to reach a wide variety of participants with different demographics characteristics and from different locations in Italy, the questionnaire was disseminated through the web. Cyclists associations' websites, Facebook groups, and forums were found using keywords (i.e., "cycling" "bicycle" "cyclists' association") on Google and on Facebook's search engine. Facebook groups with less than 500 participants were discarded. We contacted in total 45 Facebook groups and 29 websites. To reach the selected targets two methods were used: (a) firstly, the link to the questionnaire was directly posted on Facebook groups' walls or on websites bulletin boards if available; (b) secondly, an email was written to the website administrators, kindly asking to advertise the questionnaire directly on their website, through their social media channels or inside their newsletter. The second method was the one that ensured more respondents.

\section{Participants}

A total of 395 participants responded the questionnaire. After considering only those participants that had filled out the items for age, gender and that acknowledged to use the bicycle at least once a week, the remaining sample comprised 264 (67.1\%) participants. From 
these, $170(64.4 \%)$ were men, $91(34.5 \%)$ were women and $3(1.1 \%)$ did not feel identified with any of these categories. The age of the participants ranged from 19 to 82 years old. The mean for women was $40.67(S D=14.08)$, the mean for men was $46.64(S D=13.66)$, whereas the general mean value was $44.65(S D=14.05)$.

Among these participants, $29(11.0 \%)$ of them used the bicycle once a week, $28(10.6 \%)$ used it twice, other $30(11.4 \%)$ participants cycled three times a week, $26(9.8 \%)$ did so four times, $39(14.8 \%)$ of them cycled five times a week and the remaining $112(42.4 \%)$ participants used the bicycle six or more times per week. Moreover, regarding the frequency of use in comparison with other means of transportation, $31.4 \%$ of the participants reported to use the bicycle as a primary mode of transportation.

Regarding near crash experiences, 97 (36.7\%) participants had not suffered any near crash during the last year, whereas, $70(26.5 \%)$ had experienced one, $42(15.9 \%)$ two, $20(7.6 \%)$ three, and $35(13.3 \%)$ had experienced at least four near crashes. The majority of the participants had experienced a crash in their lifetime, from which 104 (39.4\%) experienced a crash without injury, and $55(20.8 \%)$ did suffer an injury. The rest, 103 (39.0\%), had not experienced any crash. Two $(0.8 \%)$ values were missing.

\section{Measures}

\section{Unsafe Cycling Behavior}

In order to measure unsafe cycling behaviors, an 18-item self-reported questionnaire was used. The items of the questionnaire were based on the type of violations and errors measured in the Driver Behavior Questionnaire (DBQ; Reason et al., 1990) and the Adolescent Cycling Behavior Questionnaire (ACBQ; Feenstra et al., 2011), adapting the former ones to the context of cycling. Moreover, five items measuring behaviors related to the use of smartphones while cycling were added. Participants were asked to state the frequency with which they undertook the type of behaviors mentioned in the items by using a 5-point Likert-type 
scale (ranging from $1=$ never to $5=$ always). Table 1 shows the item and subscale structure of the questionnaire, as well as some descriptive and reliability values. As it can be seen there, the items of the three subscales feature Smartphone-specific violations, Generic violations, and Errors.

\section{Near Crashes and Crashes}

To obtain a measure of crashes and near crashes, we used two items. Regarding the item measuring near crashes: 'In this past year, have you been about to get involved in an accident (either with other road users or a single crash) while you were using your bike?' $(0=N o$, it never happened to me, 1=Once, 2=Twice, 3=Three times, 4=Four or more). The item measuring crashes was: 'In your whole life, have you ever had an accident (either with other road users or a single crash) while you were driving your bike? $(1=$ No, it never happened to me, 2=Yes, but I did not get hurt, $3=Y e s, I$ got injured and I went to emergency services to get checked, 4=Yes, I got injured and after being checked I got hospitalized). To finally obtain three categories, the last two replies were merged into one category that represented accidents involving injuries.

\section{Statistical Analysis}

We conducted the analyses using SPSS version 23 and Mplus 6 (Muthén \& Muthén, 2010). Analysis of the data was split into several stages. First, group comparisons and correlation coefficients among the key variables were calculated. Second, we employed path analysis to test mediations, as well as direct effects, because it allowed us to estimate a model that constrains several direct effects to zero (e.g., an eventual direct effect of smartphonespecific violations on crashes), thereby, letting us test our hypotheses without the need of testing a saturated model (Hayes, 2013). Provided that two endogenous variables of our model (i.e., near crashes and crashes) are ordinal, we applied Bayesian analysis (Skrondal, \& Rabe-Hesketh, 2005). This type of analysis allows us to estimate the lower and upper values 
(also known as Credibility Intervals) within which, with a pre-defined probability, the parameter can be found given the observed data (Zyphur \& Oswald, 2013). In other words, once the parameter and the credibility intervals are obtained, one can state that there is an established probability that such parameter is comprised within the credibility interval.

\section{Results}

Table 1 displays the subscale items of the unsafe cycling behaviors questionnaire along with their mean and standard deviation values. As it can be seen, the generic violation reported as the most frequent was "Going the wrong way in a one-way street" whereas the least frequent one was “Cycle after having drunk alcohol”. With regard to smartphonespecific violations and errors, the behaviors reported as most frequent were "Use the cell/Smartphone to respond a call" and "Abruptly break in order to avoid /dodge a vehicle" respectively.

\section{[Insert Table 1]}

\section{Age and Gender Differences in Unsafe Behaviors}

Given the scarce research on cycling safety in the Italian context, we explored the relationship between the demographics age and gender in unsafe behaviors as well as in crashes and near crashes. Providing that the normal distribution assumption of all our dependent variables per gender were violated (i.e., K-S test, $p<.05$ ), we ran the MannWhitney test to explore differences between male and female in unsafe behaviors, crashes and near crashes. We did not find gender differences for Errors $(U=6636.00, z=-1.90$, $p=.057)$, Smartphone-specific violations $(\mathrm{U}=7205.00, z=-0.93 p=.351)$ and Generic violations $(\mathrm{U}=7436.00, z=-0.51, p=.606)$. Nonetheless, we found differences in gender near crashes and crashes. Concretely, we found men (Mean Rank = 138.01) to report higher frequency of near crashes than women (Mean Rank $=117.91 ; \mathrm{U}=6544.00, z=-2.13$, 
$p=.033, r=-.13)$. Moreover, consequences related to crashes were more severe for men $($ Mean Rank $=140.18)$ than for women $($ Mean Rank $=110.89 ; \mathrm{U}=5885.00, z=-3.21, p=.001$, $r=0.20)$. In these cases, using the convert equation described by Rosenthal (1991) in which the $\mathrm{z}$-score is divided by the total number of participants, it is possible to calculate the effect size, $r$ value.

In addition, we found age to be positively correlated with crashes, $r(261)=.176$, $\mathrm{p}=.004$, and negatively correlated with Errors, $r(263)=-.162, p=.008$, Generic violations, $r$ $(263)=-.356, p<.001$, and Smartphone-specific violations, $r(263)=-.409, p<.001$.

\section{Unsafe Cycling Behaviors Effect on Near Crashes and Crashes}

Table 2 displays the bivariate correlations between the key variables studied as well as the descriptive statistics. The three kinds of unsafe cycling behaviors were significantly correlated among them $(p<.01)$. Moreover, the variable Near Crashes was also significantly associate with Errors $(p<.01)$, Generic violations $(p<.05)$ and Crashes $(p<.01)$.

\section{[Insert Table 2]}

Regarding the hypothesized model, Figure 2 shows the Bayesian estimates for each path. Errors predicted near crashes (hypothesis 1), whereas Generic (hypothesis 2) and Smartphone-specific violations (hypothesis 3) did not. In turn, near crashes predicted actual crashes (hypothesis 5). Moreover, Smartphone-specific violations did predict Errors (hypothesis 4). Mediation analysis showed that near crashes were mediating the effect of Errors on crashes (Bayesian estimate $=0.250,95 \%$ CI $0.118-0.407$; hypothesis 6 ). Furthermore, Smartphone-specific violations predicted crashes throughout its consecutive 
effects on Errors and near crashes (Bayesian estimate $=0.019,95 \%$ CI $0.003-0.045$; hypothesis 7).

[Insert Figure 2]

\section{Discussion}

The aims of the current study were to examine the impact of three types of unsafe cycling behaviors (i.e., errors, generic violations, and smartphone-specific violations) on the likelihood of near crashes as well as the indirect effect of such behaviors on actual crashes among Italian cyclists. Moreover, it also aimed to analyze age and gender differences both in these behaviors, crashes, and near crashes.

It is important to note that, differently from previous studies, our findings focused on Smartphone-specific violations as a different unsafe behavior subtype. The rationale for this was that, as previously explained, such type of violations was thought to increase error occurrence by its effect on visual detection and perception. In addition, we wanted to examine whether such behaviors were indeed predicting errors and near crashes or, due to eventual compensatory behaviors (Goldenbeld et al., 2012), they were not associated.

Gender differences were found in near crashes and crashes, but not in unsafe cycling behaviors. These findings differ from those of Feenstra et al. (2011) in which boys were more prone to committing errors and common violations. Moreover, correlation analysis showed age to be positively correlated with crashes and negatively correlated with each one of the unsafe cycling behaviors, implying that older people are more likely to be involved in accidents, but less prone to engage in unsafe cycling behaviors. These findings contrast with those of Twisk et al. (2015) who reported a positive correlation between age and errors and violations in one group of adolescent participants and another negative correlation between age and crashes in the other group. Moreover, Feenstra et al. (2011) also found older participants to report higher 
frequency of exceptional violations. No difference in errors was found in their study either. Nonetheless, it is important to note that previous studies were focusing on adolescent participants, whereas our study comprises cyclists whose ages range from 19 to 82. Differences between our findings and previous ones could be due to the fact that risk taking increases from childhood to adolescence due to an increase in sensation seeking and it decreases when approaching adulthood due to the development of self-regulatory capacities (Steinberg, 2008). This way, there might be a tendency to increase engagement in unsafe behaviors during the adolescence, as found by Feenstra et al. (2011), but a decrease during adulthood, such as previous research has found (Bernhoft, \& Carstensen, 2008). Further research needs to be carried out to understand eventual age differences for each type of unsafe behavior.

Path analyses confirmed all the hypotheses except for 2 and 3. Errors predicted near crashes, and these crashes. Moreover, results confirm a mediation effect which explains the effect of smartphone-specific violations on crashes throughout errors and near crashes. These findings differ from those of Feenstra et al. (2011) according to which both errors and violations (common and exceptional) were directly predicting near crashes. In our study, only errors predicted near crash frequency. Moreover, they found exceptional violations to predict accident severity and errors to predict accident frequency, whereas we did not find significant correlations between any unsafe cycling behaviors and crashes. Twisk et al. (2015) found errors, but not violations themselves, to predict crashes, thus concurring with our findings. Nevertheless, it is worth noting two main differences between these previous studies and our research. First, we conducted the study among adults and not adolescents, thus exploring age differences in this population. And secondly, our study was set in Italy whereas theirs were carried out in The Netherlands.

This study has several theoretical and practical implications. To begin with, we have introduced smartphone-specific violations in the model and conceptualized them as a 
qualitatively different type of violation that is affecting unsafe behaviors relying on information processing (i.e., errors). Moreover, we have found them to predict near crashes and crashes through an indirect effect. This entails that smartphone-specific violations have an effect on other unsafe behaviors and, therefore, offers a broader understanding of how such behaviors end up leading to eventual crashes. In addition, given the high frequency of smartphone use reported in several studies (e.g., Goldenbeld et al., 2012; Ichikawa \& Nakahara, 2008), we want to emphasize the importance of future study of these type unsafe behaviors and how they might be contributing to errors occurrence. Moreover, adopting the Safety Pyramid model, we found near crashes to predict crashes. This provides further knowledge about how crash events might unfold and should be considered in future research.

There are some limitations to this study. We used a self-reported questionnaire to measure both unsafe cycling behaviors and safety outcomes (i.e., near crashes and crashes). This entails two limitations: (1) memories of crashes and near crashes (e.g., Chapman \& Underwood, 2000), as well as those of unsafe behaviors that do not depend on conscious control (i.e., errors), may not be accurate according to previous findings (Twisk et al., 2015; Bradburn, Rips, \& Shevell, 1987); moreover, (2) Common Method Variance (CMV), which refers to the amount of variance attributable to the use of the same method to measure related variables, constitutes a limitation to our study given that we measured all the variables using self-reported questionnaires. Moreover, we measured past crash and near crash experiences, and present behaviors. This is more likely to entail an effect of the past experiences on behaviors than vise versa (Feenstra, 2011).

Future research should address how cycling exposure may condition the results found and the present model. Nevertheless, since exposure might vary depending on the season and period of the year (and of life), its study at the individual level should take into account such individual variations. 
Our findings suggest that smartphone-specific violations appear to contribute to the frequency of errors while cycling. Furthermore, both errors and smartphone-specific violations predict crashes throughout an indirect effect on near crashes. Finally, these findings contribute to examine possible cultural factors that can moderate the relationship between unsafe cycling behaviors and crash risk.

\section{Acknowledgements}

The authors acknowledge the collaboration of Filippo Fassina, Pietro Marenco, and Ivan Oliviero. 


\section{References}

Bernhoft, I. M., \& Carstensen, G. (2008). Preferences and behaviour of pedestrians and cyclists by age and gender. Transportation Research Part F: Traffic Psychology and Behaviour, 11(2), 83-95.

Bradburn, N. M., Rips, L. J., \& Shevell, S. K. (1987). Answering Autobiographical Questions: The Impact of Memory and Inference on Surveys. Science, 236, 157-161. http://dx.doi.org/10.1126/science.3563494

Chapman, P., \& Underwood, G. (2000). Forgetting Near-Accidents: The Roles of Severity, Culpability and Experience in the Poor Recall of Dangerous Driving Situations. Applied Cognitive Psychology, 14, 31-44. http://dx.doi.org/10.1002/(SICI)10990720(200001)14:1<31::AID-ACP622>3.0.CO;2-9

DeWaard, D., Edlinger, K., \& Brookhuis, K. (2011). Effects of listening to music, and of using a handheld and handsfree telephone on cycling behavior. Transportation Research Part F, 14, 626-637. http://dx.doi.org/10.1016/j.trf.2011.07.001

DeWaard, D., Lewis-Ewans, B., Jelijs, B., Tucha, O., \& Brookhuis, K. (2014). The effects of operating a touch screen smartphone and other common activities performed while bicycling on cycling behavior, Transportation Research Part F, 20, 196-206. http://dx.doi.org/10.1016/j.trf.2013.12.003

DeWaard, D., Schepers, P., Ormel, W., \& Brookhuis, K. (2010). Mobile phone use while cycling: Incidence and effects on behaviour and safety. Ergonomics, 53(1), 30-42. doi:10.1080/00140130903381180 
DeWaard, D., Westerhuis, F., \& Lewis-Evans, B. (2015). More screen operating than calling: The results of observing cyclists' behaviors while using mobile phones. Accident Analysis and Prevention, 76, 42-48. http://dx.doi.org/10.1016/j.aap.2015.01.004

De Winter, J. C., \& Dodou, D. (2010). The Driver Behaviour Questionnaire as a predictor of accidents: A meta-analysis. Journal of safety research, 41(6), 463-470. http://dx.doi.org/10.1016/j.jsr.2010.10.007

ERSO European Road Safety Observatory. (2015a). Road Safety Country Overview - Italy. Retrieved from http://ec.europa.eu/transport/road_safety/specialist/erso/pdf/country_overviews/dacot a-country-overview-it_en.pdf

ERSO European Road Safety Observatory. (2015b). Traffic Safety Basic Facts 2015 Cyclists. Retrieved from http://ec.europa.eu/transport/road_safety/pdf/statistics/dacota/bfs2015_cyclists.pdf

Feenstra, H., Ruiter, R. A., Schepers, J., Peters, G. J., \& Kok, G. (2011). Measuring risky adolescent cycling behaviour. International journal of injury control and safety promotion, 18(3), 181-187. http://dx.doi.org/10.1080/17457300.2010.540334

Goldenbeld, C., Houtenbos, M., Ehlers, E., \& DeWaard, D. (2012). The use and risk of portable electronic devices while cycling among different groups. Journal of Safety Research, 43, 1-8. http://dx.doi.org/10.1016/j.jsr.2011.08.007

Hayes, A. F., (2013). Multiple Mediator Models. In F. A. Hayes (Ed.), Introduction to Mediation, Moderation and Conditional Process Analysis (pp. 123-164). New York, USA: The Guilford Press.

Hollingworth, M. A., Harper, A.J., \& Hamer, M. (2015). Risk factors for cycling accident related injury: The UK Cycling for Health Survey. Journal of Transport \& Health, 2, 189-194. http://dx.doi.org/10.1016/j.jth.2015.01.001 
ISTAT Italian National Institute of Statistics. (2015). Rapporto anno 2014, incidenti stradali in Italia. Retrieved from http://www.istat.it/it/files/2015/11/Incidentistradali2014.pdf?title=Incidenti+stradali+in+Italia+-+03\%2Fnov\%2F2015++ Testo+integrale.pdf

Ichikawa, M., \& Nakahara, S. (2008). Japanese High School Students' Usage of Mobile Phones While Cycling. Traffic Injury Prevention, 9(1), 42-47. http://dx.doi.org/10.1080/15389580701718389

Jeffs, L., Berta, W., Lingard, L., \& Baker, G. R. (2012). Learning from near misses: from quick fixes to closing off the Swiss-cheese holes. BMJ quality \& safety, 21(4), 287294. doi: 10.1136/bmjqs-2011-000256. http://dx.doi.org/10.1136/bmjqs-2011-000256 Jones, S., Kirchsteiger, C., \& Bjerke, W. (1999). The importance of near miss reporting to further improve safety performance. Journal of Loss Prevention in the process industries, 12(1), 59-67. http://dx.doi.org/10.1016/S0950-4230(98)00038-2

Muthén, L. K., \& Muthén, B. O. (2010). Mplus user's guide, 6. Retrieved from https://www.statmodel.com/download/usersguide/Mplus\%20Users\%20Guide\%20v6. pdf

Phimister, J. R., Oktem, U., Kleindorfer, P. R., \& Kunreuther, H. (2003). Near-miss incident management in the chemical process industry. Risk Analysis,23(3), 445-459. http://dx.doi.org/10.1111/1539-6924.00326

Powell, N. B., Schechtman, K. B., Riley, R. W., Guilleminault, C., Chiang, R. P. Y., \& Weaver, E. M. (2007). Sleepy driver near-misses may predict accident risks. SLEEP, $30(3), 331-342$.

Reason, J. (1990). Human Error. Cambridge: Cambridge University Press. http://dx.doi.org/10.1017/CBO9781139062367 
Reason, J., Manstead, A., Stradling, S., Baxter, J., \& Campbell, K. (1990). Errors and violations on the roads: a real distinction? Ergonomics, 33(10/11), 1315-1332. http://dx.doi.org/10.1080/00140139008925335

Rosenthal, R. (1991). Meta-analytic procedures for social research ( $2^{\text {nd }}$ ed.). Newbury Park, CA: Sage. http://dx.doi.org/10.4135/9781412984997

Rowe, R., Roman, G. D., McKenna, F. P., Barker, E., \& Poulter, D. (2015). Measuring errors and violations on the road: A bifactor modeling approach to the Driver Behavior Questionnaire. Accident Analysis \& Prevention, 74, 118-125. http://dx.doi.org/10.1016/j.aap.2014.10.012

Runyan, C. W. (1998). Using the Haddon matrix: introducing the third dimension. Injury Prevention, 4, 302-307.

Sakashita, C., Senserrick, T., Lo, S., Boufous, S., Rome, L., D., \& Ivers, R. (2014). The Motorcycle Rider Behavior Questionnaire: Psychometric properties and application amongst novice riders in Australia. Transportation Research Part F, 22, 126-139. http://dx.doi.org/10.1016/j.trf.2013.10.005

Saleh, J. H., Saltmarsh, E. A., Favarò, F. M., \& Brevault, L. (2013). Accident precursors, near misses, and warning signs: Critical review and formal definitions within the framework of Discrete Event Systems. Reliability Engineering \& System Safety, 114, 148-154. http://dx.doi.org/10.1016/j.ress.2013.01.006

Skrondal, A., \& Rabe-Hesketh, S. (2005). Structural Equation Modeling: Categorical Variables. In B. S. Everitt \& D. C. Howell (Eds.), Encyclopedia of Statistics in Behavioral Sciences, volume 4 (pp. 1905-1910). Chichester, UK: John Willey and Sons, Ltd. http://dx.doi.org/10.1002/0470013192.bsa596 
Steinberg, L. (2008). A social neuroscience perspective on adolescent risk-taking. Developmental review, 28(1), 78-106.

Terzano, K. (2013). Bicycling safety and distracted behavior in The Hague, the Netherlands. Accident Analysis and Prevention, 57, 87-90. http://dx.doi.org/10.1016/j.aap.2013.04.007

Turner, C., \& McClure, R. (2003). Age and gender differences in risk-taking behaviour as an explanation for high incidence of motor vehicle crashes as a driver in young males. Injury control and safety promotion, 10(3), 123-130. http://dx.doi.org/10.1076/icsp.10.3.123.14560

Twisk, D. A., Commandeur, J. J., Vlakveld, W. P., Shope, J. T., \& Kok, G. (2015). Relationships amongst psychological determinants, risk behaviour, and road crashes of young adolescent pedestrians and cyclists: Implications for road safety education programmes. Transportation Research Part F: Traffic Psychology and Behaviour, 30, 45-56. http://dx.doi.org/10.1016/j.trf.2015.01.011

Wegman, F., Zhang, F., \& Dijkstra, A. (2012). How to make more cycling good for road safety? Accident Analysis \& Prevention, 44(1), 19-29. http://dx.doi.org/10.1016/j.aap.2010.11.010

Wright, L., \& Van der Schaaf, T. (2004). Accident versus near miss causation: a critical review of the literature, an empirical test in the UK railway domain, and their implications for other sectors. Journal of Hazardous Materials, 111(1), 105-110. http://dx.doi.org/10.1016/j.jhazmat.2004.02.049

Zyphur, M. J., \& Oswald, F. L. (2015). Bayesian Estimation and Inference: A User's Guide. Journal of Management, 41(2), 390-420. http://dx.doi.org/10.1177/0149206313501200 
